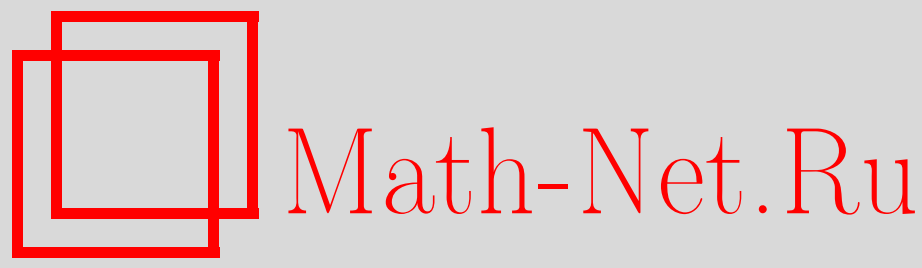

Н. Т. Йылмаз, Алгебраическое интегрирование полевых уравнений сигма-модели, ТМФ, 2009, том 159, номep 2, 266-282

DOI: https://doi.org/10.4213/tmf6348

Использование Общероссийского математического портала Math-Net.Ru подразумевает, что вы прочитали и согласны с пользовательским соглашением http://www . mathnet.ru/rus/agreement

Параметры загрузки:

IP : 54.224 .60 .19

26 апреля 2023 г., $12: 34: 23$

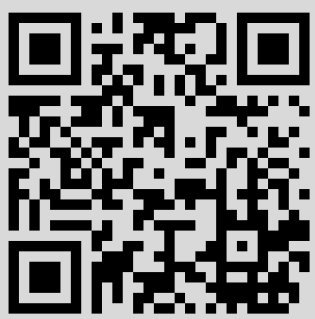




\title{
ФИЗИКА
}

Том 159, № 2

май, 2009

2009 г.

\author{
Н. Т. Йылмаз
}

\section{АЛГЕБРАИЧЕСКОЕ ИНТЕГРИРОВАНИЕ ПОЛЕВЫХ УРАВНЕНИЙ СИГМА-МОДЕЛИ}

Доказано, что дуализованная алгебра сигма-модели с симметрическим косетпространством является алгеброй Ли. Показано, что она порождает подходящее присоединенное представление, которое позволяет локально интегрировать полевые уравнения, в результате чего получаются уравнения первого порядка.

Ключевые слова: сигма-модели, формулировки первого порядка, дуализованная алгебра.

\section{1. ВВЕДЕНИЕ}

Полевой состав сигма-модели с симметрическим косет-пространством дается скалярными полями, которые параметризуют таргет-многообразие, являющееся однородным римановым глобально симметричным пространством. Удваивая состав полей за счет введения дуальных полей высших порядков, можно реализовать теорию с помощью конструкции расширенного косета. Происхождение этого метода связано с дуализацией теорий супергравитации [1], [2], скалярные секторы которой соответствуют упомянутым типам сигма-моделей. Наиболее важным элементом расширенной реализации этой теории является конструкция дуализованной косетной параметризующей алгебры, которая в случае чистой сигма-модели является деформацией исходной косет-алгебры. Хотя геометрическая конструкция этой расширенной формулировки пока еще не очень хорошо известна, дуализованная алгебра для общей косетной сигма-модели с симметрическим таргет-пространством найдена в работах [3], [4]. В этих работах в рамках метода дуализации также получены полевые уравнения первого порядка как условия согласованности вложения. Однако ни одна из цитированных работ на дает прямой алгебраической связи этих уравнений первого порядка с уравнениями второго порядка, которые возникают из принципа наименьшего действия.

В настоящей работе мы дадим строгое доказательство того, что полученная в работах [3], [4] дуализованная косет-алгебра действительно является алгеброй Ли. Наше доказательство главным образом будет заключаться в том, чтобы показать,

${ }^{*}$ Department of Mathematics and Computer Science, Çankaya University, Ankara, Turkey. E-mail: ntyilmaz@cankaya.edu.tr 
что в наиболее общих терминах (для произвольной сигма-модели) коммутационные соотношения дуализованной алгебры, которая возникает как деформация обычной косет-алгебры, удовлетворяют тождествам Якоби. Следуя этому, мы также обсудим, что дуализованная косет-алгебра, являясь алгеброй Ли, допускает естественное присоединенное представление исходной косет-алгебры, которая является ее подалгеброй Ли. Наконец, мы покажем, что, предполагая это естественное присоединенное представление, порожденное дуализованной алгеброй, можно локально алгебраически связать уравнения первого порядка, полученные в работах [3], [4], с полевыми уравнениями второго порядка. А именно, исходя из анзаца уравнения первого порядка и применяя внешнюю производную, мы покажем, что возникают полевые уравнения второго порядка в специальном представлении, порожденном дуализованной косет-алгеброй.

В разделе 2, который носит достаточно формальный характер, исследуются все возможные условия в дуализованной косет-алгебре общего вида и таким образом дается полное доказательство структуры ее алгебры Ли для произвольной косетной сигма-модели. В разделе 3 обсуждается естественное присоединенное представление исходной косет-алгебры, предложенное в рамках данной схемы. В разделе 4 доказано, что уравнения первого порядка из работ [3], [4] действительно являются истинными локальными уравнениями, которые можно получить из полевых уравнений второго порядка путем локального устранения внешней производной, когда выбрано указанное специальное представление.

\section{2. ДУАЛИЗОВАННАЯ КОСЕТ-АЛГЕБРА}

Дуализованная косет-алгебра сигма-модели с общим симметрическим пространством найдена в работах [3], [4]. Она порождена набором генераторов

$$
\left\{H_{i}, E_{\alpha}, \widetilde{H}_{i}, \widetilde{E}_{\alpha}\right\}
$$

где первые два набора генераторов соответствуют подмножеству базиса КартанаВейля группы глобальных симметрий лагранжиана сигма-модели, который порождает разрешимую подалгебру Ли $s$. Здесь при $i=1,2, \ldots, r$ генераторы $H_{i}$ образуют подмножество картановских генераторов алгебры Ли группы глобальных симметрий сигма-модели, а $E_{\alpha}$ порождают корневое подпространство $\Delta_{\mathrm{nc}}^{+}$некомпактных положительных корней [3], [4]. Последние два набора генераторов (2.1) дуальны предыдущим. Коммутационные соотношения дуализованной косет-алгебры, порожденной генераторами (2.1), можно записать как

$$
\begin{aligned}
& {\left[H_{i}, H_{j}\right]=\left[H_{i}, \widetilde{H}_{j}\right]=\left[E_{\alpha}, \widetilde{H}_{j}\right]=\left[\widetilde{E}_{\alpha}, \widetilde{H}_{j}\right]=\left[\widetilde{H}_{i}, \widetilde{H}_{j}\right]=\left[\widetilde{E}_{\alpha}, \widetilde{E}_{\beta}\right]=0,} \\
& {\left[H_{i}, E_{\alpha}\right]=\alpha_{i} E_{\alpha}, \quad\left[E_{\alpha}, E_{\beta}\right]= \begin{cases}N_{\alpha, \beta} E_{\alpha+\beta}, & \alpha+\beta \in \Delta, \\
0, & \alpha+\beta \notin \Delta,\end{cases} } \\
& {\left[E_{\alpha}, \widetilde{E}_{\alpha}\right]=\frac{1}{4} \sum_{j=1}^{r} \alpha_{j} \widetilde{H}_{j}, \quad\left[H_{j}, \widetilde{E}_{\alpha}\right]=-\alpha_{j} \widetilde{E}_{\alpha},}
\end{aligned}
$$




$$
\left[E_{\alpha}, \widetilde{E}_{\beta}\right]=\left\{\begin{array}{lll}
0, & \alpha-\beta \notin \Delta & \text { или } \quad \alpha-\beta \in \Delta, \\
N_{\alpha,-\beta} \widetilde{E}_{\gamma}, & \alpha-\beta \in \Delta, \quad \beta-\alpha \in \Delta_{\mathrm{nc}}^{+} \quad \text { и } \quad \alpha-\beta=-\gamma \notin \Delta_{\mathrm{nc}}^{+},
\end{array}\right.
$$

где $\Delta$ соответствует корням алгебры Ли группы глобальных симметрий сигма-модели, $\alpha_{i}$ - компоненты корневого вектора, а вещественные коэффициенты $N_{\alpha \beta}$ являются структурными константами, соответствующими коммутационным соотношениям корневых подпространств генераторов $E_{\alpha}$. Следует заметить, что если $\alpha$ и $\beta$ являются некомпактыми положительными корнями и $\alpha+\beta \in \Delta$, то $\alpha+\beta-$ также некомпактный положительный корень. Действительно, если это не так, то

$$
\left[E_{\alpha}, E_{\beta}\right]=N_{\alpha, \beta} E_{\alpha+\beta} \notin s,
$$

что противоречит замыканию разрешимой подалгебры Ли. Введем обозначения

$$
\left\{T_{m}\right\} \equiv\left\{H_{i}, E_{\alpha}\right\}, \quad \widetilde{T}_{m} \equiv\left\{\widetilde{H}_{i}, \widetilde{E}_{\alpha}\right\}
$$

так что область определения индекса $m$ расщепляется на два множества:

$$
m=\overbrace{1, \ldots, r}^{i, j, k, \ldots}, \underbrace{r+1, r+2, \ldots, \operatorname{dim} s}_{\alpha, \beta, \gamma, \ldots} .
$$

Другими словами,

$$
\begin{aligned}
T_{1} & =H_{1}, & T_{2} & =H_{2}, & \ldots, & T_{r}=H_{r}, \\
\widetilde{T}_{1} & =\widetilde{H}_{1}, & \widetilde{T}_{2} & =\widetilde{H}_{2}, & \ldots, & \widetilde{T}_{r}=\widetilde{H}_{r}, \\
T_{r+1} & =E_{\alpha}, & T_{r+2} & =E_{\beta}, & \ldots, & \\
\widetilde{T}_{r+1} & =\widetilde{E}_{\alpha}, & \widetilde{T}_{r+2} & =\widetilde{E}_{\beta}, & \ldots . &
\end{aligned}
$$

Коммутационные соотношения (2.2) компактнее можно записать в виде

$$
\begin{aligned}
& {\left[H_{i}, H_{j}\right]=0, \quad\left[H_{i}, E_{\alpha}\right]=\alpha_{i} E_{\alpha}, \quad\left[E_{\alpha}, E_{\beta}\right]= \begin{cases}0, & \alpha+\beta \notin \Delta, \\
N_{\alpha, \beta} E_{\alpha+\beta}, & \alpha+\beta \in \Delta,\end{cases} } \\
& {\left[\widetilde{T}_{m}, \widetilde{T}_{n}\right]=0, \quad\left[E_{\gamma}, \widetilde{T}_{m}\right]=\tilde{f}_{\gamma m}^{n} \widetilde{T}_{n}, \quad\left[H_{j}, \widetilde{T}_{m}\right]=\widetilde{g}_{j m}^{n} \widetilde{T}_{n},}
\end{aligned}
$$

где матрицы структурных констант $\tilde{f}_{\gamma}$ и $\widetilde{g}_{j}$ в расщепленном виде можно представить каK

$$
\begin{aligned}
& 1,2, \ldots, r \quad \alpha, \beta, \ldots, \gamma, \ldots
\end{aligned}
$$

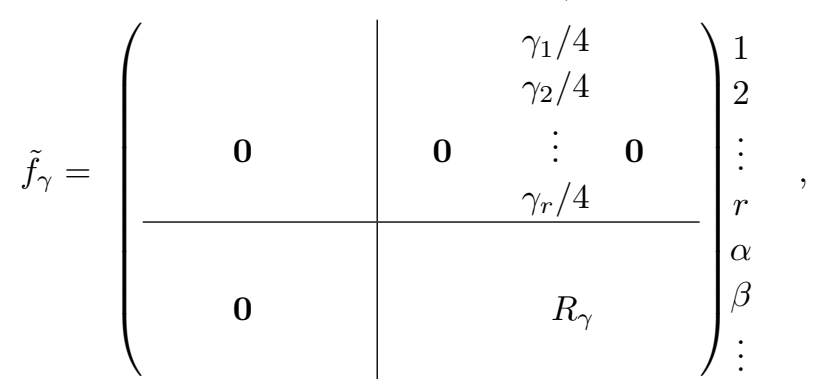




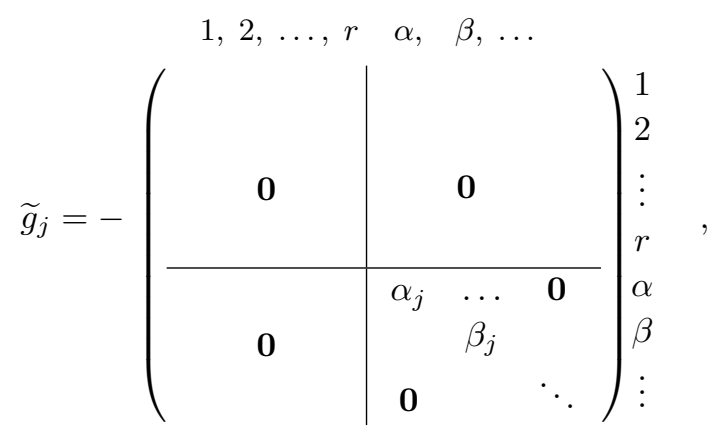

где матрица $R_{\gamma}$ в правом нижнем блоке (2.7) определена как

$$
\left(R_{\gamma}\right)_{\kappa}^{\tau}=\left\{\begin{array}{ll}
N_{\gamma,-\kappa}, & \kappa-\tau=\gamma, \\
0, & \kappa-\tau \neq \gamma,
\end{array} \quad \tau, \kappa=\alpha, \beta, \ldots\right.
$$

Заметим, что выражения типа $\kappa-\tau=\gamma$ здесь и далее следует понимать как равенства для корневых векторов.

Сформулируем некоторые свойства матрицы $R_{\gamma}$.

1. Если для фиксированного столбца $\kappa$ выполнено $\gamma-\kappa \notin \Delta$ или $\gamma-\kappa \in \Delta$, но $\gamma-\kappa \neq-\tau$ для любой строки $\tau$, то $\kappa-$ нулевой столбец (все его элементы равны нулю).

2. Если для фиксированного столбца $\kappa$ выполнено $\gamma-\kappa \in \Delta$, то для строк $\tau$ и $\tau^{\prime}$, определяемых из $\gamma-\kappa=-\tau$ и $\gamma-\kappa=-\tau^{\prime}$, должно быть выполнено $\tau=\tau^{\prime}$. Другими словами, если столбец $\kappa$ ненулевой, то в нем имеется только один ненулевой элемент $N_{\gamma,-\kappa}$.

3. Если для фиксированной строки $\tau$ не существует такого столбца $\kappa$, что $\gamma-\kappa=-\tau$, то строка $\tau$ нулевая (все ее элементы равны нулю). Другими словами, если $\gamma+\tau \notin \Delta$, то не существует такого $\kappa$, что $\gamma-\kappa=-\tau$, и строка $\tau$ является нулевой. Однако если $\gamma+\tau \in \Delta$, то из нашего предыдущего обсуждения следует, что $\gamma+\tau \in \Delta_{\text {nc }}^{+}$, т.е. существует такой столбец $\kappa$, что $\gamma-\kappa=-\tau$, и строка $\tau$ имеет ненулевой элемент.

4. Для фиксированной строки $\tau$ и для таких столбцов $\kappa$ и $\kappa^{\prime}$, что $\gamma-\kappa=-\tau$ и $\gamma-\kappa^{\prime}=-\tau$, должно быть выполнено $\kappa=\kappa^{\prime}$, другими словами, если строка $\tau$ ненулевая, то в ней имеется только один ненулевой элемент $N_{\gamma,-\kappa}$.

5. Следует отметить, что п. 1 и 3 непротиворечивы, т.е. если $\gamma-\kappa \neq-\tau$, то это же верно как для столбцов, так и для строк.

6. Для диагональных элементов $\tau=\kappa$ из условия $\gamma-\kappa=-\tau$ вытекает, что $\gamma=$ $\kappa-\tau=0 \notin \Delta$. Поскольку $\gamma \in \Delta$, условие $\gamma-\kappa=-\tau$ не может быть выполнено для диагональных элементов, поэтому все диагональные элементы должны равняться нулю.

7. Для $n$ столбцов, когда $\kappa$ пробегает $\Delta_{\mathrm{nc}}^{+}$, если $\gamma-\kappa \notin \Delta$ или $\gamma-\kappa \in \Delta$, но $\gamma-\kappa \neq-\tau$ для любого $\tau \in \Delta_{\text {nс }}^{+}$, то имеются $n$ нулевых столбцов. Поскольку в каждой строке и каждом столбце имеется по крайней мере один ненулевой элемент, должны существовать $\operatorname{dim} \Delta_{\mathrm{nc}}^{+}-n$ ненулевых элементов в $\operatorname{dim} \Delta_{\mathrm{nc}}^{+}-n$ различных столбцах и строках. Это означает, что имеются также $n$ нулевых строк. 
8. Если $\gamma+\tau \notin \Delta$ для $n$ строк, а $\tau$ при этом пробегает $\Delta_{\text {nc }}^{+}$, то имеются $n$ нулевых строк. Рассуждая, как в п. 7, получаем $n$ нулевых столбцов. Поскольку верное для строк верно также для столбцов, п. 7 и 8 также непротиворечивы.

В итоге все диагональные элементы матрицы $R_{\gamma}$ равны нулю, каждая строка и каждый столбец содержит не более одного ненулевого элемента, причем число нулевых строк равно числу нулевых столбцов.

Хотя в работах [3] и [4] в результате стандартного метода дуализации выведены структурные константы алгебры (2.2), ни в одной из этих работ не доказано, что определяемая в (2.2) алгебра, которую можно назвать дуализирующей деформацией косет-алгебры ${ }^{1)}$ рассматриваемой сигма-модели, образует алгебру Ли. Поэтому в этом разделе мы докажем, что алгебра, определенная в (2.2), действительно является алгеброй Ли. В общем случае $m$-мерная алгебра Ли порождается $m$ генераторами $X_{a}$, для которых

$$
\left[X_{a}, X_{b}\right]=C_{a b}^{c} X_{c}, \quad C_{a b}^{c}=-C_{b a}^{c}, \quad\left[X_{a}, X_{a}\right]=0 .
$$

Генераторы должны также удовлетворять тождеству Якоби

$$
\left[X_{a},\left[X_{b}, X_{c}\right]\right]+\left[X_{b},\left[X_{c}, X_{a}\right]\right]+\left[X_{c},\left[X_{a}, X_{b}\right]\right]=0 .
$$

Таким образом, наша задача состоит в том, чтобы показать, что генераторы (2.1), структурные константы которых определены в (2.2), удовлетворяют тождеству Якоби (2.11). Во-первых, если выбрать $X_{a}=T_{m}, X_{b}=T_{n}, X_{c}=T_{l}$, тождество (2.11) выполняется непосредственно, поскольку базис $\left\{T_{m}\right\}$ порождает алгебру Ли, которая является разрешимой подалгеброй Ли в алгебре Ли группы глобальных симметрий лагранжиана сигма-модели [3], [4].

Во-вторых, если выбрать $X_{a}=\widetilde{T}_{m}, X_{b}=\widetilde{T}_{n}, X_{c}=\widetilde{T}_{l}$, то из (2.6) получаем

$$
\left[\widetilde{T}_{m}, 0\right]+\left[\widetilde{T}_{n}, 0\right]+\left[\widetilde{T}_{l}, 0\right]=0,
$$

что также выполнено. Если положить $X_{a}=T_{l}, X_{b}=\widetilde{T}_{m}, X_{c}=\widetilde{T}_{n}$, то снова из (2.6) имеем

$$
\left[T_{l}, 0\right]-U_{l n}^{t}\left[\widetilde{T}_{m}, \widetilde{T}_{t}\right]+U_{l m}^{t}\left[\widetilde{T}_{n}, \widetilde{T}_{t}\right]=0,
$$

что выполняется в силу коммутации дуальных генераторов. Согласно последнему равенству имеем

$$
\left[T_{l}, \widetilde{T}_{m}\right]=U^{t}{ }_{l m} \widetilde{T}_{t},
$$

где структурные константы $U^{t}{ }_{l m}$ определяются из (2.6).

Наконец, рассмотрим $X_{a}=T_{l}, X_{b}=T_{n}, X_{c}=\widetilde{T}_{m}$. В этом случае из (2.11) после некоторых преобразований находим

$$
Z^{t}{ }_{l n} U^{s}{ }_{t m}=\left(U_{l} U_{n}-U_{n} U_{l}\right)^{s}{ }_{m},
$$

где мы положили

$$
\left[T_{l}, T_{n}\right]=Z^{t}{ }_{l n} T_{t}
$$

и ввели матрицу структурных констант $\left(U_{l}\right)^{s}{ }_{m}=U^{s}{ }_{l m}$.

\footnotetext{
1)Эта косет-алгебра является разрешимой подалгеброй Ли группы глобальных симметрий сигма-модели.
} 
Теперь докажем, что (2.15) выполнено в трех различных случаях:

1) $T_{l}=H_{j}, T_{n}=H_{k}$;

2) $T_{l}=H_{j}, T_{n}=E_{\gamma}$

3) $T_{l}=E_{\gamma}, T_{n}=E_{\lambda}$.

В первом случае $\left[H_{j}, H_{k}\right]=0$ и, таким образом, левая часть $(2.15)$ обращается в нуль. В силу (2.14) и (2.6) правая часть (2.15) принимает вид

$$
\left(\widetilde{g}_{j} \widetilde{g}_{k}-\widetilde{g}_{k} \widetilde{g}_{j}\right)_{m}^{s}
$$

Однако из (2.8) имеем

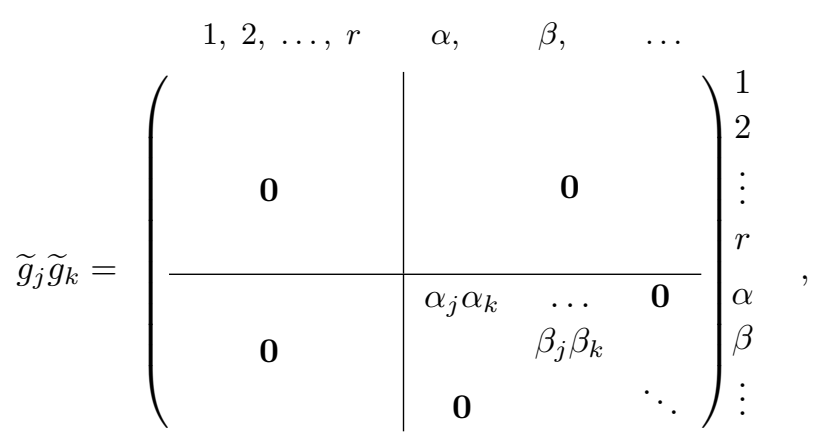

аналогично для $\widetilde{g}_{k} \widetilde{g}_{j}$. Таким образом, подставляя (2.18) в (2.17), видим, что правая часть (2.15) также обращается в нуль. Для второго случая соотношение (2.15) принимает вид

$$
Z^{t}{ }_{j \gamma} U^{s}{ }_{t m}=\left(U_{j} U_{\gamma}-U_{\gamma} U_{j}\right)^{s}{ }_{m}
$$

Преобразуем (2.20) с учетом отождествлений

$$
\begin{array}{lll}
Z_{j \gamma}^{i}=0, & Z_{j \gamma}^{\beta}=0, & \text { если } \quad \beta \neq \gamma, \\
Z_{j \gamma}^{\gamma}=\gamma_{j}, & U_{j}=\widetilde{g}_{j}, & U_{\gamma}=\tilde{f}_{\gamma} .
\end{array}
$$

Тогда доказываемое матричное равенство принимает вид

$$
\gamma_{j} \tilde{f}_{\gamma}=\widetilde{g}_{j} \tilde{f}_{\gamma}-\tilde{f}_{\gamma} \widetilde{g}_{j}
$$

Левая часть данного равенства равна

$$
\begin{aligned}
& 1,2, \ldots, r \quad \alpha, \beta, \ldots, \gamma, \ldots
\end{aligned}
$$

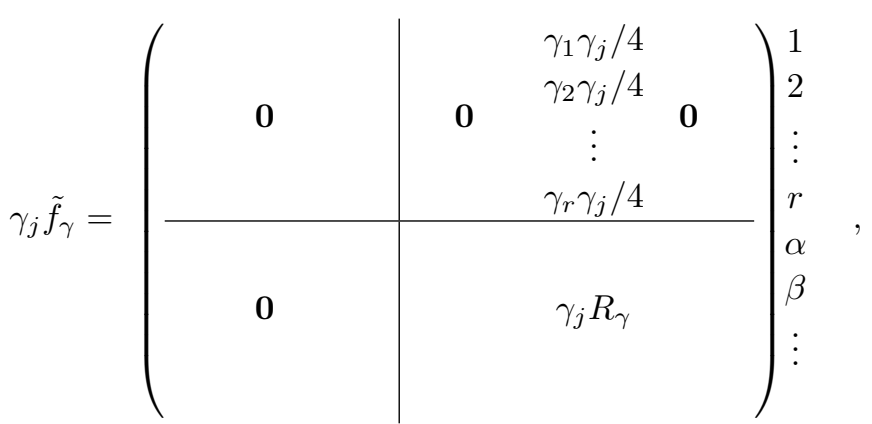

где $\gamma_{j} R_{\gamma}$ - произведение скаляра $\gamma_{j}$ на матрицу $R_{\gamma}$, заданную в $(2.9)$. 
Для вычисления правой части (2.21) из (2.7) и (2.8) сначала находим $1,2, \ldots, r \quad \alpha, \beta, \ldots$
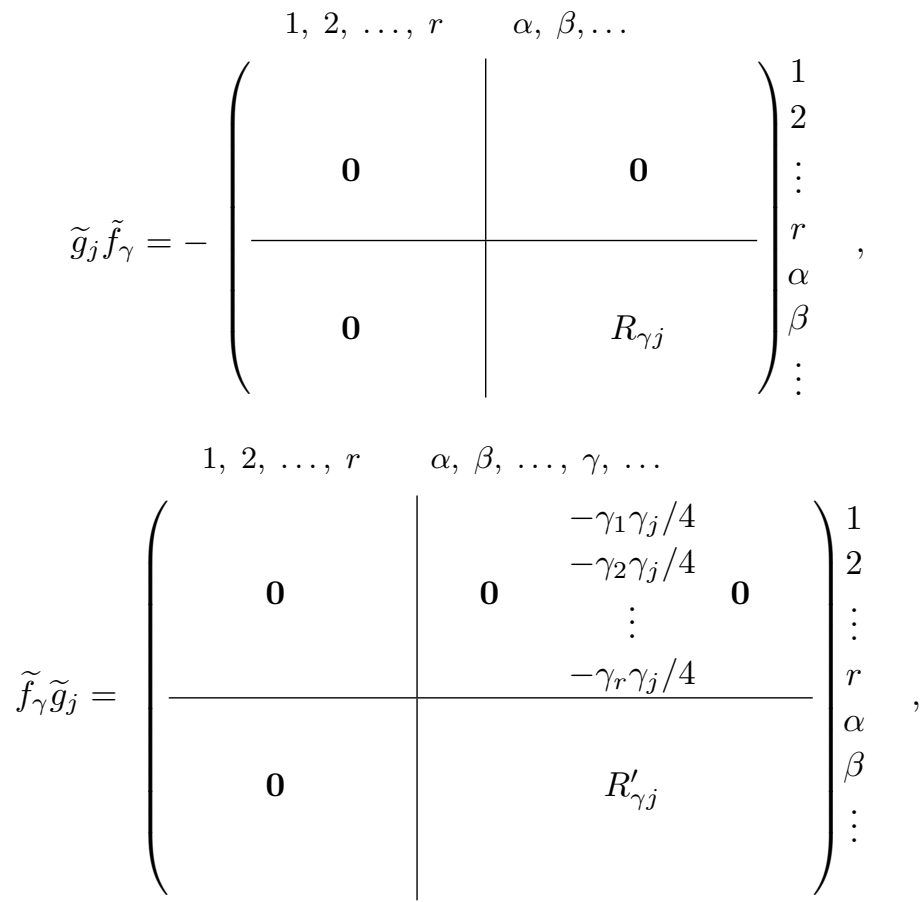

где введены матрицы

$$
\begin{aligned}
& \left(R_{\gamma j}\right)_{\kappa}^{\tau}=\left\{\begin{array}{ll}
-\tau_{j} N_{\gamma,-\kappa}, & \kappa-\tau=\gamma, \\
0, & \kappa-\tau \neq \gamma,
\end{array} \quad \tau, \kappa=\alpha, \beta, \ldots,\right. \\
& \left(R_{\gamma j}^{\prime}\right)_{\kappa}^{\tau}=\left\{\begin{array}{ll}
-\kappa_{j} N_{\gamma,-\kappa}, & \kappa-\tau=\gamma, \\
0, & \kappa-\tau \neq \gamma,
\end{array} \quad \tau, \kappa=\alpha, \beta, \ldots\right.
\end{aligned}
$$

(еще раз напомним, что равенство $\kappa-\tau=\gamma$ следует понимать как равенство корневых векторов; подчеркнем, что $\tau_{j}$ и $\kappa_{j}$ являются компонентами этих векторов). Матрица $R_{\gamma}$ определена в (2.9). Очевидно, что ненулевые элементы матриц $R_{\gamma j}$ и $R_{\gamma j}^{\prime}$ находятся в строках и столбцах с теми же номерами, что у ненулевых элементов матрицы $\gamma_{j} R_{\gamma}$. Поэтому правая часть (2.21) принимает вид

$$
\begin{aligned}
& 1,2, \ldots, r \quad \alpha, \beta, \ldots, \gamma, \ldots
\end{aligned}
$$

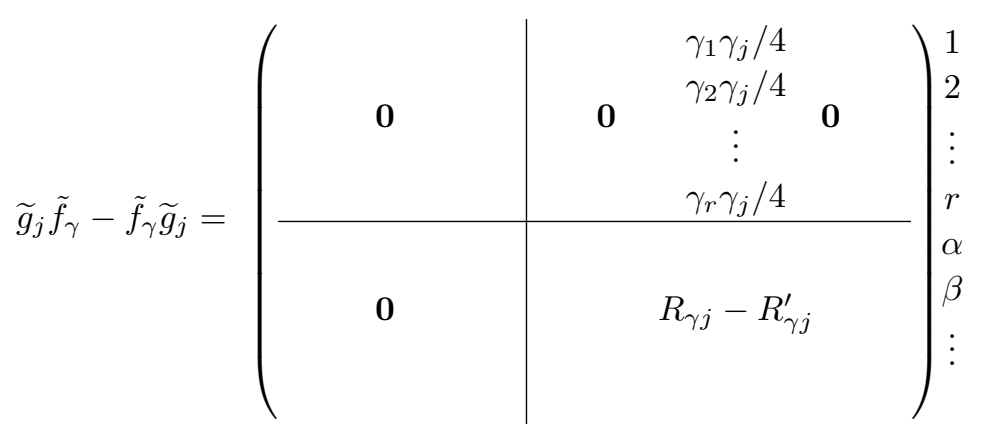


С одной стороны, при сравнении (2.22) и (2.27) видно, что три блока, кроме одного (в нижнем правом углу), равны друг другу. С другой стороны, поскольку ненулевые элементы матриц $\gamma_{j} R_{\gamma}, R_{\gamma j}$ и $R_{\gamma j}^{\prime}$ находятся в идентичных местах, нам надо показать, что эти элементы в правом нижнем блоке в левой части и в правой части формулы (2.21) равны. В матрице $R_{\gamma j}-R_{\gamma j}^{\prime}$ ненулевые элементы в строке $\tau$ и столбце $\kappa$ имеют вид

$$
-N_{\gamma,-\kappa} \tau_{j}+N_{\gamma,-\kappa} \kappa_{j}=N_{\gamma,-\kappa}\left(\kappa_{j}-\tau_{j}\right) .
$$

Однако из (2.9) следует, что ненулевой элемент существует тогда и только тогда, когда $\gamma-\kappa=-\tau$. Это корневое условие верно также для компонент корневого вектора, т.е. $\kappa_{j}-\tau_{j}=\gamma_{j}$. Таким образом, в матрице $R_{\gamma j}-R_{\gamma j}^{\prime}$ ненулевые элементы в строке $\tau$ и столбце $\kappa$ принимают вид $N_{\gamma,-\kappa} \gamma_{j}$ и совпадают с ненулевыми элементами в правом нижнем блоке матрицы (2.22), стоящей в левой части (2.21).

Наконец, в третьем случае $\left(T_{l}=E_{\gamma}\right.$ и $\left.T_{n}=E_{\lambda}\right)$ из (2.15) получаем матричное равенство

$$
N_{\gamma \lambda} \tilde{f}_{\gamma+\lambda}=\tilde{f}_{\gamma} \tilde{f}_{\lambda}-\tilde{f}_{\lambda} \tilde{f}_{\gamma}
$$

Чтобы получить это равенство, мы положили

$$
Z_{\gamma \lambda}^{i}=0, \quad Z_{\gamma \lambda}^{\beta}=0, \quad \text { если } \quad \gamma+\lambda \neq \beta, \quad Z_{\gamma \lambda}^{\gamma+\lambda}=N_{\gamma \lambda}, \quad U_{\gamma}=\tilde{f}_{\gamma} .
$$

Следует отметить, что если $\gamma+\lambda$ не является корнем, то левая часть равенства (2.29) равна нулю. Если эта сумма является корнем, то, как мы видели выше, она должна лежать в $\Delta_{\text {nc }}^{+}$, и в этом случае левая часть (2.29) принимает вид

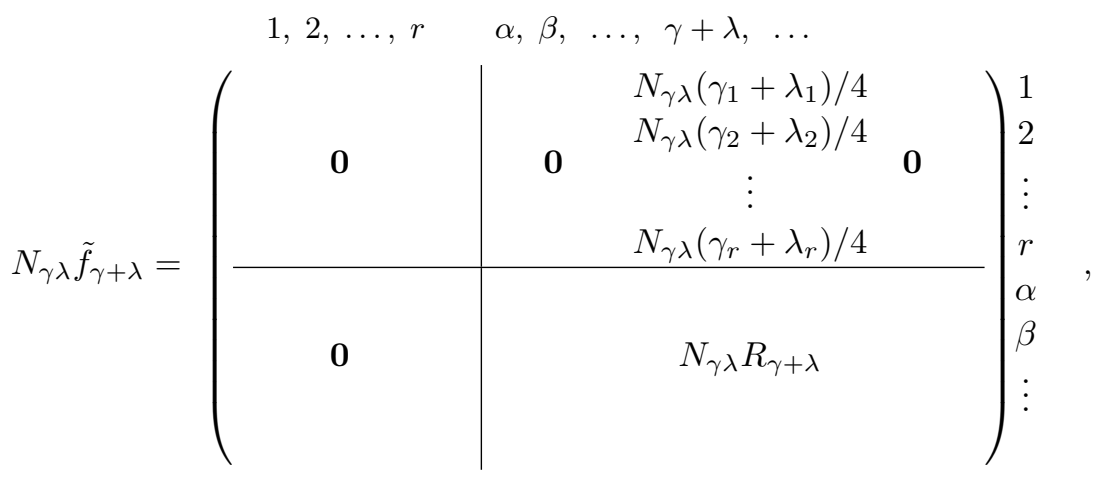

где матрица $R_{\gamma+\lambda}$ (которая в правом нижнем блоке умножена на структурную константу $\left.N_{\gamma \lambda}\right)$ определена как

$$
\left(R_{\gamma+\lambda}\right)_{\kappa}^{\tau}=\left\{\begin{array}{ll}
N_{\gamma+\lambda,-\kappa}, & \kappa-\tau=\gamma+\lambda, \\
0, & \kappa-\tau \neq \gamma+\lambda,
\end{array} \quad \tau, \kappa=\alpha, \beta, \ldots .\right.
$$

4 Теоретическая и математическая физика, т. 159, № 2, 2009 г. 
Теперь после матричного умножения с учетом (2.7) получаем

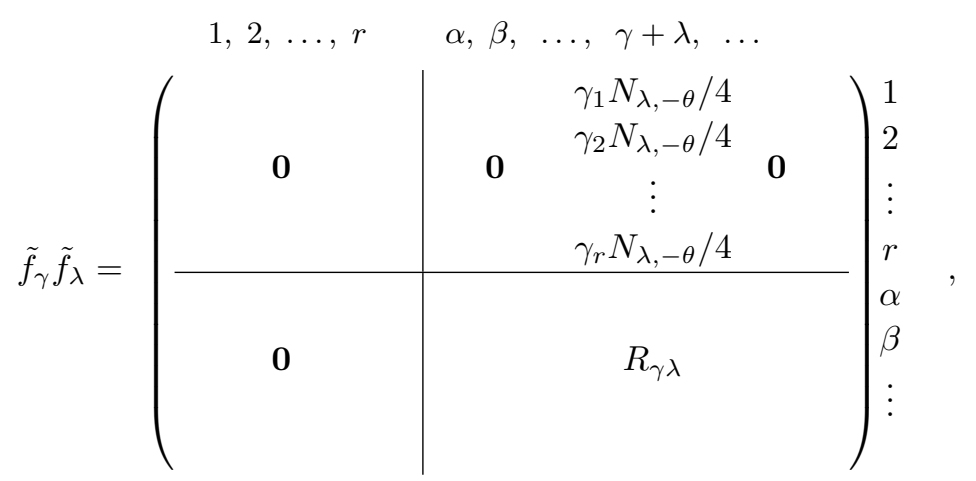

где $\theta=\gamma+\lambda$. Если $\gamma+\lambda$ не является корнем, то верхний правый блок в (2.33) равен нулю, поскольку в этом случае не будет столбца $\theta$ в $R_{\lambda}$, который удовлетворял бы условию $\theta=\gamma+\lambda$, так что строка $\gamma$ из $R_{\lambda}$ будет нулевой. В (2.33) мы имеем матрицу

$$
R_{\gamma \lambda}=R_{\gamma} R_{\lambda}
$$

Используя определение $(2.9)$ и свойства матриц $R_{\gamma}$, можно явно вычислить $R_{\gamma \lambda}$ как

$$
\left(R_{\gamma \lambda}\right)^{\tau}{ }_{\kappa}=\left(R_{\gamma} R_{\lambda}\right)^{\tau}{ }_{\kappa}=\sum_{v=\alpha, \beta, \ldots}\left(R_{\gamma}\right)_{v}^{\tau}\left(R_{\lambda}\right)^{v}{ }_{\kappa} .
$$

Здесь используются свойства матрицы $R_{\gamma}$, перечисленные после (2.9). Матрица $R_{\gamma \lambda}$ обладает теми же свойствами, что и $R_{\gamma}$, за исключением конкретных значений ненулевых элементов и их расположения: все ее диагональные элементы равны нулю, каждая строка и каждый столбец содержит не более одного ненулевого элемента, причем число нулевых строк равно числу нулевых столбцов. Для элемента из $R_{\gamma \lambda}$ номер его строки определяется таким образом: сначала находим $v$ из условия $\kappa-v=\lambda$ на ненулевой элемент матрицы $R_{\lambda}$, а затем находим номер строки $\tau$ из условия $v-\tau=\gamma$ для матрицы $R_{\gamma}$. Номер столбца находится аналогично. Разумеется, если равенство $\kappa-v=\lambda$ не выполняется ни при каких $v$, то мы имеем нулевые строку и столбец в $R_{\lambda}$, а также нулевой столбец $\kappa$ в $R_{\gamma \lambda}$. Если равенство $v-\tau=\gamma$ не выполняется ни при каких $\tau$, то мы имеем нулевые строку и столбец в $R_{\gamma}$, и столбец $\kappa$ в $R_{\gamma \lambda}$ также нулевой. В обоих случаях при этом в $R_{\gamma \lambda}$ имеется также нулевая строка.

Теперь можно записать правую часть (2.29) как

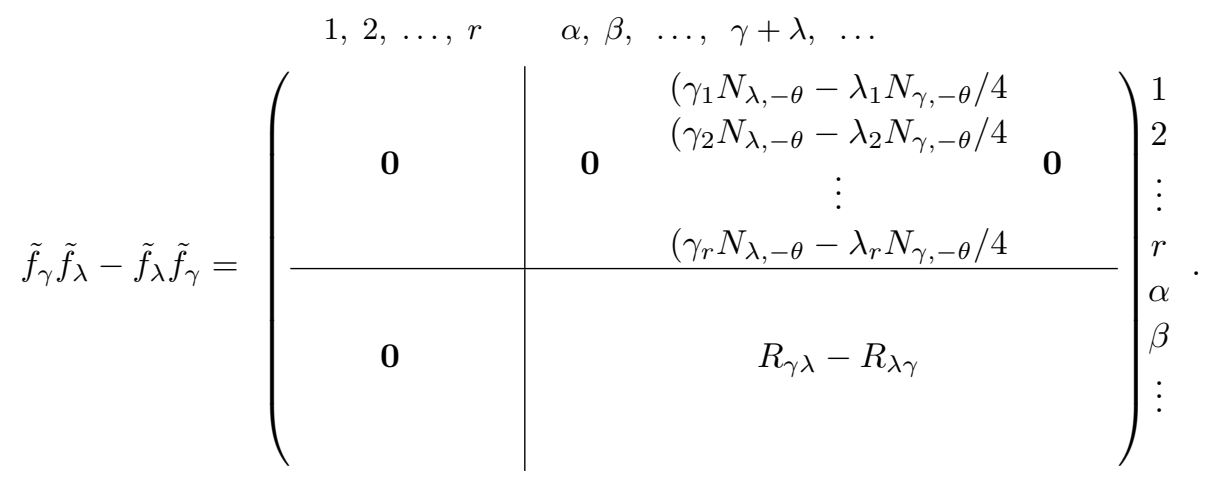


Прежде чем двигаться дальше, покажем, что ненулевые элементы в матрицах $R_{\gamma \lambda}$ и $R_{\lambda \gamma}$ находятся на одних и тех же местах. В матрице $R_{\gamma \lambda}$ в строке $\tau$ и столбце $\kappa$ имеется ненулевой элемент, если и только если для некоторого $v$ выполнено

$$
v-\tau=\gamma, \quad \kappa-v=\lambda
$$

для некоторого $\xi$. Складывая вновь левые и правые части двух последних равенств, получаем условие на ненулевой элемент в $R_{\gamma \lambda}$ в виде $\kappa-\tau=\gamma+\lambda$ (при этом $\gamma+\lambda+\tau$ несомненно является элементом из $\Delta_{\mathrm{nc}}^{+}$). Если в $R_{\lambda \gamma}$ в строке $\tau^{\prime}$ и столбце $\kappa^{\prime}$ имеется ненулевой элемент, то

$$
\xi-\tau^{\prime}=\lambda, \quad \kappa^{\prime}-\xi=\gamma
$$

Складывая левые и правые части данных равенств, получаем условие на ненулевой элемент в $R_{\gamma \lambda}$ в виде $\kappa^{\prime}-\tau^{\prime}=\gamma+\lambda$. Поскольку, очевидно, $\gamma+\lambda=\lambda+\gamma$, множество пар $(\tau, \kappa)$, удовлетворяющих условию на ненулевой элемент для $R_{\gamma \lambda}$, совпадает с множеством пар $\left(\tau^{\prime}, \kappa^{\prime}\right)$, удовлетворяющих условию на ненулевой элемент для $R_{\lambda \gamma}$. Другими словами, ненулевые элементы этих двух матриц занимают одни и те же места. Следовательно, матрицы $R_{\gamma \lambda}$ и $R_{\lambda \gamma}$ имеют одно и то же количество нулеых строк и нулевых стлолбцов, которые к тому же расположены одинаково. Заметим также, что эти свойства указанных двух матриц очевидным образом совпадают со свойствами матрицы $R_{\gamma+\lambda}$.

Напомним, что матрицы $R_{\gamma+\lambda}$ и $R_{\gamma \lambda}-R_{\lambda \gamma}$ - это правые нижние блоки в левой и правой частях равенства (2.29) соответственно. Но сначала мы рассмотрим ненулевые элементы в правом верхнем блоке (2.36) в столбце $\gamma+\lambda$. Эти элементы имеют вид

$$
\frac{\gamma_{i} N_{\lambda,-\theta}-\lambda_{i} N_{\gamma,-\theta}}{4}
$$

Как уже отмечалось, если $\gamma+\lambda$ не является корнем, то верхний правый блок в $(2.36)$ будет нулевым, совпадая при этом с верхним правым блоком в левой части (2.29), который равен нулю, поскольку $N_{\gamma \lambda}=0$. Однако если $\gamma+\lambda$ является корнем, то, поскольку косет-алгебра замкнута, имеем $\gamma+\lambda \in \Delta_{\text {nc }}^{+}$. Тогда существует такой корень $\theta=\gamma+\lambda \in \Delta_{\text {nc }}^{+}$, что $\lambda-\theta=-\gamma$ и $\gamma-\theta=-\lambda$. Если сумма трех корневых генераторов базиса Картана-Вейля равна нулю, скажем $\alpha+\beta+\gamma=0$, то

$$
N_{\alpha \beta}=N_{\beta \gamma}=N_{\gamma \alpha}
$$

Поскольку для ненулевых элементов (2.39) $\gamma+\lambda-\theta=0$, имеем $N_{\lambda,-\theta}=N_{\gamma \lambda}$ и $N_{\gamma,-\theta}=-N_{-\theta, \gamma}=-N_{\gamma \lambda}$. Таким образом, ненулевые элементы из (2.39) принимают вид

$$
\frac{\gamma_{i} N_{\lambda,-\theta}-\lambda_{i} N_{\gamma,-\theta}}{4}=\frac{N_{\gamma \lambda}\left(\gamma_{i}+\lambda_{i}\right)}{4}
$$

и равны ненулевым элементам из верхнего правого блока в $(2.31)$ в столбце $\gamma+\lambda$.

Теперь рассмотрим равенство правых нижних блоков в левой и правой частях (2.29). Определим значения ненулевых элементов матрицы $R_{\gamma \lambda}-R_{\lambda \gamma}$. Необходимо рассмотреть два случая: $\gamma+\lambda$ является корнем и $\gamma+\lambda$ не является корнем. 
Пусть сначала $\gamma+\lambda$ - не корень. Тогда из (2.11) непосредственно следует, что левая часть (2.29) равна нулю. Рассмотрим правую часть. Для строки $\tau$ и столбца $\kappa$ в $R_{\gamma \lambda}-R_{\lambda \gamma}$, если оба условия (2.37) и (2.38) выполнены и тем самым элемент существует, он должен быть равен $N_{\lambda,-\kappa} N_{\gamma,-v}-N_{\gamma,-\kappa} N_{\lambda,-\xi}$. Однако для существования этого элемента из условий (2.37) и (2.38), а также из тождества (2.40) имеем условия

$$
N_{\gamma,-v}=N_{\tau \gamma}, \quad N_{\lambda,-\xi}=N_{\tau \lambda} .
$$

Таким образом, этот элемент принимает вид

$$
N_{\lambda,-\kappa} N_{\tau \gamma}-N_{\gamma,-\kappa} N_{\tau \lambda}
$$

В общем случае, если для четырех корней выполняется равенство $\alpha+\beta+\gamma+$ $\delta=0$ и сумма ни одной из пар не равна нулю, то структурные константы базиса Картана-Вейля удовлетворяют тождеству

$$
N_{\alpha \beta} N_{\gamma \delta}+N_{\beta \gamma} N_{\alpha \delta}+N_{\gamma \alpha} N_{\beta \delta}=0 .
$$

Поскольку, как уже было показано, $\kappa-\tau=\gamma+\lambda$, имеем $\gamma+\lambda+\tau-\kappa=0$. С учетом (2.44) получаем

$$
N_{\gamma \lambda} N_{\tau,-\kappa}+N_{\lambda \tau} N_{\gamma,-\kappa}+N_{\tau \gamma} N_{\lambda,-\kappa}=0
$$

Однако, в силу того что $\gamma+\lambda$ не является корнем, $N_{\gamma \lambda}=0$, и мы получаем

$$
-N_{\tau \lambda} N_{\gamma,-\kappa}+N_{\tau \gamma} N_{\lambda,-\kappa}=0
$$

где левая часть в точности равна (2.43). Это доказывает равенство правых нижних блоков в левой и правой частях $(2.29)$ в случае, если $\gamma+\lambda$ не является корнем. Заметим, ранее уже было доказано, что либо оба условия (2.37) и (2.38) выполняются, либо оба этих условия не выполняются, т.е. ненулевые элементы матриц $R_{\gamma \lambda}$ и $R_{\lambda \gamma}$ расположены на одних и тех же местах.

Пусть теперь $\gamma+\lambda$ - корень. Как мы уже заметили, ненулевые строки и столбцы в матрице $R_{\gamma+\lambda}$ и матрицах $R_{\gamma \lambda}$ и $R_{\lambda \gamma}$ совпадают, кроме того, ненулевые элементы в $R_{\gamma+\lambda}, R_{\gamma \lambda}$ и $R_{\lambda \gamma}$ стоят на одних и тех же местах. Теперь надо показать, что в матричной разности $R_{\gamma \lambda}-R_{\lambda \gamma}$ разности ненулевых элементов не обращаются в нуль и, более того, равны соответствующим ненулевым элементам из $R_{\gamma+\lambda}$. Другими словами, необходимо доказать, что если строка $\tau$ и столбец $\kappa$ содержат ненулевой элемент, то выполнено равенство

$$
N_{\gamma \lambda} N_{\gamma+\lambda,-\kappa}=N_{\lambda,-\kappa} N_{\gamma,-v}-N_{\gamma,-\kappa} N_{\lambda,-\xi}
$$

Снова используя тождество (2.40), из (2.47) получаем

$$
N_{\gamma \lambda} N_{\gamma+\lambda,-\kappa}=N_{\lambda,-\kappa} N_{\tau \gamma}-N_{\gamma,-\kappa} N_{\tau \lambda} .
$$

Теперь, поскольку $\gamma+\lambda-\kappa+\tau=0$, из (2.40) имеем $N_{\gamma+\lambda,-\kappa}=N_{-\kappa, \tau}$. Поэтому (2.48) можно записать как

$$
0=N_{\gamma \lambda} N_{\tau,-\kappa}+N_{\lambda,-\kappa} N_{\tau \gamma}+N_{\gamma,-\kappa} N_{\lambda \tau} .
$$


Это равенство выполнено в силу справедливости (2.45), что и является желаемым результатом.

Тем самым мы завершили доказательство равенства (2.29) для всех возможных случаев. Вместе с предыдущими результатами, установленными в данном разделе, это доказывает, что алгебраическая структура, заданная в (2.2) и являющаяся деформацией разрешимой подалгебры Ли группы глобальных симметрий сигма-модели, подчиняется тождеству Якоби (2.11) и тем самым определяет алгебру Ли.

\section{3. ПРИСОЕДИНЕННОЕ ПРЕДСТАВЛЕНИЕ}

Покажем, что дуализованная косет-алгебра (2.2) содержит присоединенное представление подалгебры $s$, порожденной исходными косет-генераторами $\left\{T_{m}\right\} \equiv$ $\left\{H_{i}, E_{\alpha}\right\}$. Эта подалгебра является исходной косет-алгеброй сигма-модели, которая представляет собой разрешимую подалгебру алгебры Ли группы глобальной симметрии. Указанное присоединенное представление существует в силу общей схемы $\left[\left\{T_{m}\right\},\left\{\widetilde{T}_{n}\right\}\right] \subset\left\{\widetilde{T}_{n}\right\}$ структуры дуализованной косет-алгебры (2.2). Чтобы найти это представление, определим линейное отображение

$$
f: s \longrightarrow g l(S, \mathbb{R})
$$

где $S$ - размерность подалгебры $s$. По аналогии с общим присоединенным представлением алгебры Ли общего вида предполагаем, что отображение $f$ имеет вид

$$
f\left(T_{l}\right)=U_{l},
$$

где $U_{l}-(S \times S)$-матрица с элементами $U^{t}{ }_{l m}$, которые являются определенными в (2.14) структурными константами. Линейность и свойство (3.2) определяют действие $f$ на всей подалгебре $s$. Это линейное отображение является гомоморфизмом алгебр, если

$$
f([M, N])=[f(M), f(N)]
$$

для всех $M, N \in s$. Если подставить $M=M^{l} T_{l}$ и $N=N^{k} T_{k}$ в (3.3), то видно, что (3.3) выполнено, если $f\left(\left[T_{l}, T_{n}\right]\right)=\left[f\left(T_{l}\right), f\left(T_{n}\right)\right]$. Это равенство, используя соотношения (2.16) и (3.2), а также предположение о линейности отображения, можно записать в виде

$$
Z^{t}{ }_{l n} U^{s}{ }_{t m}=\left(U_{l} U_{n}-U_{n} U_{l}\right)^{s}{ }_{m},
$$

что совпадает с тождеством Якоби (2.15), справедливость которого мы доказали в разделе 2. Таким образом, можно заключить, что отображение $f$, действие которого на базис $\left\{T_{m}\right\}$ определяется согласно (3.2), есть гомоморфизм алгебр и образует $(S \times S)$-матричное представление косет-алгебры $s$, которая является подалгеброй в (2.2). Дуализованная косет-алгебра (2.2), которая является деформацией своей подалгебры $s$ и структура алгебры Ли которой установлена в разделе 2 , порождает естественное присоединенное представление исходной косет-алгебры $s$. 


\section{4. СИГМА-МОДЕЛЬНЫЕ ПОЛЕВЫЕ УРАВНЕНИЯ ПЕРВОГО ПОРЯДКА}

Теперь покажем, что представление, установленное в разделе 3 , позволяет вывести полевые уравнения первого порядка для сигма-модели на симметрическом пространстве. Полевые уравнения первого порядка сигма-модели с таргет-многообразием косета в виде симметрического пространства впервые появились в работах [3], [4] как результат дуализации косет-конструкции этих теорий. Однако в указанных работах они были формально введены как условия согласованности в рамках дуализирующей теории. Здесь мы алгебраически докажем, что они соответствуют уравнениям, которые можно получить локальным интегрированием полевых уравнений второго порядка. Другими словами, мы покажем, что если выбрать представление, указанное в разделе 3 , то можно получить полевые уравнения второго порядка в сигма-модели с симметрическим пространством, если взять внешнюю производную от уравнений первого порядка, полученных в работах [3], [4]. Таким образом, мы используем систему уравнений из работ [3], [4]:

$$
* \vec{\Psi}=(-1)^{D} e^{\boldsymbol{\Gamma}} e^{\boldsymbol{\Lambda}} \overrightarrow{\mathbf{A}}
$$

где $D$ - размерность базисного многообразия, $S$ - размер вектор-столбцов $\overrightarrow{\boldsymbol{\Psi}}, \overrightarrow{\mathbf{A}}$, которые имеют компоненты

$$
\begin{array}{ll}
\mathbf{\Psi}^{i}=\frac{1}{2} d \phi^{i}, & i=1,2, \ldots, r, \\
\mathbf{\Psi}^{\alpha+r}=e^{\alpha_{i} \phi^{i} / 2} \boldsymbol{\Omega}_{\gamma}^{\alpha} d \chi^{\gamma}, & \alpha=1,2, \ldots, S-r, \\
\mathbf{A}^{i}=\frac{1}{2} d \tilde{\phi}^{i}, & i=1,2, \ldots, r, \\
\mathbf{A}^{\alpha+r}=d \widetilde{\chi}^{\alpha}, & \alpha=1,2, \ldots, S-r .
\end{array}
$$

Здесь $\phi^{i}$ и $\chi^{\gamma}$ - скалярные поля, относительно которых надо найти решение и которые параметризуют косет-пространство таргет-многообразия сигма-модели. Отметим, что $\alpha$ обозначает и некомпактные положительные корни, и их соответствующую нумерацию. Кроме того, $\tilde{\phi}^{i}$ и $\widetilde{\chi}^{\alpha}$ являются произвольными $(D-2)$-формами, которые возникают из дуализации косет-отображения в рамках дуализованной косет-реализации теории. В $(4.1) \boldsymbol{\Gamma}\left(\phi^{i}\right)$ и $\boldsymbol{\Lambda}\left(\chi^{\beta}\right)$ суть $(S \times S)$-матричные функционалы с компонентами

$$
\boldsymbol{\Gamma}_{n}^{k}=\frac{1}{2} \phi^{i} \widetilde{g}_{i n}^{k}, \quad \boldsymbol{\Lambda}_{n}^{k}=\chi^{\alpha} \tilde{f}_{\alpha n}^{k},
$$

где матрицы $\tilde{f}_{\alpha}$ и $\widetilde{g}_{i}$ определены соответственно в (2.7) и (2.8). Рассматривая определения (2.6) и (2.14) под действием присоединенного представления (3.1) алгебры $s$, существование которого доказано в разделе 3 , получаем отождествление

$$
e^{\boldsymbol{\Gamma}} e^{\boldsymbol{\Lambda}}=e^{\phi^{i} \widetilde{g}_{i} / 2} e^{\chi^{\alpha} \tilde{f}_{\alpha}} \equiv e^{\phi^{i} H_{i} / 2} e^{\chi^{\alpha} E_{\alpha}}=\nu,
$$

где $\nu$ - косет-представитель сигма-модели [3], [4]. Таким образом, с учетом представления, определенного в (3.1), под действием которого

$$
H_{i} \longrightarrow \widetilde{g}_{i}, \quad E_{\alpha} \longrightarrow \tilde{f}_{\alpha},
$$


систему уравнений первого порядка (4.1) можно записать в виде

$$
* \vec{\Psi}=(-1)^{D} \nu \overrightarrow{\mathbf{A}}
$$

Возьмем теперь внешнюю производную обеих частей:

$$
d(* \vec{\Psi})=(-1)^{D} d \nu \overrightarrow{\mathbf{A}}
$$

где использовалось, что $d \mathbf{A}^{m}=0$. Поскольку (4.6) является векторным уравнением, его можно записать как ${ }^{2)} \overrightarrow{\mathbf{A}}=(-1)^{D} \nu^{-1} * \overrightarrow{\mathbf{\Psi}}$. Подставляя это равенство в (4.7), получаем

$$
d(* \vec{\Psi})=d \nu \nu^{-1} * \vec{\Psi} .
$$

Из этого уравнения сразу видно, что $\mathcal{G}=d \nu \nu^{-1}$ является формой Картана, индуцированной косет-отображением $\nu$. Она была вычислена в явном виде в работе [4] и может быть записана как

$$
\mathcal{G}=\frac{1}{2} d \phi^{i} H_{i}+e^{\beta_{i} \phi^{i} / 2} \boldsymbol{\Omega}_{\alpha}^{\beta} d \chi^{\alpha} E_{\beta},
$$

где

$$
\boldsymbol{\Omega}=\sum_{m=0}^{\infty} \frac{\omega^{m}}{(m+1) !}=\left(e^{\omega}-I\right) \omega^{-1} .
$$

Здесь $(S-r) \times(S-r)$-матрица $\omega$ имеет компоненты $\omega_{\beta}^{\gamma}=\chi^{\alpha} K_{\alpha \beta}^{\gamma}$, причем $K_{\alpha \beta}^{\gamma}$ определяются как $\left[E_{\alpha}, E_{\beta}\right]=K_{\alpha \beta}^{\gamma} E_{\gamma}$.

Поскольку мы выбрали представление, порожденное отображением (3.2), можно записать (4.9) в виде

$$
\mathcal{G}=\frac{1}{2} d \phi^{i} \widetilde{g}_{i}+e^{\beta_{i} \phi^{i} / 2} \boldsymbol{\Omega}_{\alpha}^{\beta} d \chi^{\alpha} \tilde{f}_{\beta}
$$

Подставляя (4.11) в (4.8), получаем (в покомпонентной записи)

$$
d\left(* \boldsymbol{\Psi}^{m}\right)=\left(\frac{1}{2} d \phi^{i} \widetilde{g}_{i n}^{m}+e^{\beta_{i} \phi^{i} / 2} \boldsymbol{\Omega}_{\alpha}^{\beta} d \chi^{\alpha} \tilde{f}_{\beta n}^{m}\right) \wedge * \boldsymbol{\Psi}^{n} .
$$

При $1 \leqslant m \leqslant r$ это равенство дает

$$
\frac{1}{2} d\left(* d \phi^{i}\right)=\frac{1}{2} d \phi^{j} \wedge \widetilde{g}_{j n}^{i} * \Psi^{n}+e^{\beta_{k} \phi^{k} / 2} \mathbf{\Omega}_{\alpha}^{\beta} d \chi^{\alpha} \wedge \tilde{f}_{\beta n}^{i} * \Psi^{n} .
$$

В силу (2.8) первый член в правой части (4.13) обращается в нуль, а если разбить сумму во втором члене по индексу $n$, то получаем

$$
\frac{1}{2} d\left(* d \phi^{i}\right)=e^{\beta_{k} \phi^{k} / 2} \boldsymbol{\Omega}_{\alpha}^{\beta} d \chi^{\alpha} \wedge\left(\tilde{f}_{\beta j}^{i} * \Psi^{j}+\tilde{f}_{\beta, \gamma+r}^{i} * \mathbf{\Psi}^{\gamma+r}\right) .
$$

2) Заметим, что $\nu^{-1}$ существует по определению. 
Теперь в силу (2.7) первый член в правой части (4.14) снова обращается в нуль. Дальнейшее разбиение индекса в сумме по остающимся членам дает

$$
\frac{1}{2} d\left(* d \phi^{i}\right)=e^{\beta_{k} \phi^{k} / 2} \boldsymbol{\Omega}_{\alpha}^{\beta} d \chi^{\alpha} \wedge\left(\tilde{f}_{\beta, \beta+r}^{i} * \mathbf{\Psi}^{\beta+r}+\sum_{\kappa \neq \beta} \tilde{f}_{\beta, \kappa+r}^{i} * \mathbf{\Psi}^{\kappa+r}\right) .
$$

В силу (2.7) вторая сумма в правой части (4.15) также обращается в нуль. Получив 3 ) значение $\tilde{f}_{\beta, \beta+r}^{i}$ из (2.7), а также используя (4.2), окончательно получаем

$$
d\left(* d \phi^{i}\right)=\frac{1}{2} \sum_{\alpha, \beta, \gamma \in \Delta_{\mathrm{nc}}^{+}} \beta_{i} e^{\beta_{k} \phi^{k} / 2} \boldsymbol{\Omega}_{\alpha}^{\beta} d \chi^{\alpha} \wedge e^{\beta_{j} \phi^{j} / 2} \boldsymbol{\Omega}_{\gamma}^{\beta} * d \chi^{\gamma},
$$

где $i$ - свободный индекс. Система уравнений (4.16) является в точности уравнениями второго порядка дилатонного поля сигма-модели, которые были получены в работах [3]-[6].

С другой стороны, из равенства (4.12) при $m>r$ получаем

$$
d *\left(e^{\beta_{i} \phi^{i} / 2} \boldsymbol{\Omega}_{\alpha}^{\beta} d \chi^{\alpha}\right)=\left(\frac{1}{2} d \phi^{i} \widetilde{g}_{i n}^{\beta+r}+e^{\kappa_{i} \phi^{i} / 2} \boldsymbol{\Omega}_{\alpha}^{\kappa} d \chi^{\alpha} \tilde{f}_{\kappa n}^{\beta+r}\right) \wedge * \boldsymbol{\Psi}^{n},
$$

где $\beta$ - свободный индекс. Аналогично нашему вычислению, выполненному выше, и в силу (2.7) и (2.8) после исключения обращающихся в нуль членов в правой части, получаем

$$
d *\left(e^{\beta_{i} \phi^{i} / 2} \boldsymbol{\Omega}_{\alpha}^{\beta} d \chi^{\alpha}\right)=-\frac{1}{2} \beta_{i} d \phi^{i} \wedge * \Psi^{\beta+r}+e^{\kappa_{i} \phi^{i} / 2} \boldsymbol{\Omega}_{\alpha}^{\kappa} d \chi^{\alpha} \tilde{f}_{\kappa, \gamma+r}^{\beta+r} \wedge * \Psi^{\gamma+r} .
$$

Более того, используя (4.2) и (2.7), окончательно имеем

$$
\begin{aligned}
d *\left(e^{\beta_{i} \phi^{i} / 2} \boldsymbol{\Omega}_{\alpha}^{\beta} d \chi^{\alpha}\right)= & -\frac{1}{2} \beta_{i} d \phi^{i} \wedge\left(e^{\beta_{j} \phi^{j} / 2} \boldsymbol{\Omega}_{\alpha}^{\beta} * d \chi^{\alpha}\right)+ \\
& +\sum_{\kappa-\theta=-\beta} e^{\kappa_{i} \phi^{i} / 2} \boldsymbol{\Omega}_{\alpha}^{\kappa} d \chi^{\alpha} \wedge\left(N_{\kappa,-\theta} e^{\theta_{j} \phi^{j} / 2} \boldsymbol{\Omega}_{\sigma}^{\theta} * d \chi^{\sigma}\right)
\end{aligned}
$$

где в правой части во втором члене сумма берется по индексу $\kappa \in \Delta_{\text {nc }}^{+}(\beta-$ снова свободный индекс) и в силу (2.9) индекс $\theta \in \Delta_{\text {nс }}^{+}$(если соответствующий корень существует в $\Delta_{\text {nc }}^{+}$, когда фиксированы $\beta$ и $\kappa$ ) следует выбрать согласно сформулированному выше корневому условию ${ }^{4)}$. Поскольку индекс (корень) $\beta$ пробегает $\Delta_{\text {nc }}^{+}$, эти уравнения являются уравнениями второго порядка аксионного поля сигма-модели, которые были выведены в работах [3]-[6].

\footnotetext{
${ }^{3)}$ Следует обратить внимание, что первый член в скобках в правой части (4.15) является не суммой, а одним слагаемым.

4) Конечно, если такой корень $\theta$ не существует в $\Delta_{\mathrm{nc}}^{+}$, то в силу $(2.9)$ этот член для конкретного выбора $\beta$ и $\kappa$ равен нулю. Укажем также, что в (4.19) нет суммирования по индексу $\theta$, в противном случае $\theta$ фиксируется при выборе $\beta$ и $\kappa$.
} 


\section{5. ЗАКЛЮЧЕНИЕ}

Мы дали строгое доказательство того, что дуализованная алгебра косетной сигма-модели с глобально римановым симметричным таргет-пространством действительно является алгеброй Ли. Хотя коммутационные соотношения дуализованной косет-алгебры были получены в работе [4], их Ли-алгебраическая структура там не была установлена. Показав, что структурные константы или коммутаторы действительно удовлетворяют тождеству Якоби, мы обосновали понятие алгебры Ли дуализованной алгебры, которая является расширением исходной косет-алгебры сигма-модели. Мы также отметили, что дуализованная косет-алгебра, которая содержит в себе обыкновенную алгебру и поэтому может рассматриваться как ее деформация, допускает присоединенное представление исходной косет-алгебры. Наконец, мы показали, что под действием этого специального представления полевые уравнения второго порядка можно получить дифференцированием уравнений первого порядка, которые появились в работах [3], [4] как условия согласованности дуализирующей конструкции. Мы доказали, что эти условия согласованности являются истинными алгебраическими уравнениями первого порядка в соответствующей сигма-модели.

Цель настоящей работы - показать, что под действием специального представления, порожденного алгеброй дуальности, уравнения Эйлера-Лагранжа второго порядка можно проинтегрировать и получить полевые уравнения первого порядка. Эти уравнения первого порядка уже получены в работах [3], [4]. Однако только в рамках настоящей работы доказано, что они алгебраически правильны, поскольку в работах [3], [4] они возникли как условие согласованности, соответствующее дуализации теории. С другой стороны, в настоящей работе мы доказали, что если применить внешнюю производную к этим уравнениям первого порядка, то получаются правильные полевые уравнения второго порядка, которые являются уравнениями Эйлера-Лагранжа. Учитывая приведенный в настоящей работе анализ, легко утверждать, что дуализация теории помимо своей расширенной геометрической конструкции является эффективным способом поиска правильного представления косет-алгебры так, что это представление приводит к интегрированию полевых уравнений. Другими словами, в рамках дуализованной теории исходная косет-алгебра реализована как ее ли-алгебраическая деформация (дуализованная косет-алгебра) таким образом, что порождаемое присоединенное представление принимает вид подходящего представления, допускающего интегрирование полевых уравнений. Мы показали, что представление, позволяющее построить полевые уравнения первого порядка, зависит от ли-алгебраической структуры дуализованной косет-алгебры, которая является специальным и нетривиальным расширением исходной. В работах [3], [4] эта алгебра и полевые уравнения первого порядка были получены исходя из геометрической точки зрения. Здесь же, продемонстрировав наследование ли-алгебраической структуры и соответственно присоединенного представления, мы алгебраически дополнили результаты работ [3], [4]. Таким образом, наше точное доказательство дополнительно обосновывает правильность полевых уравнений первого порядка в сигма-модели с симметрическим простран- 
ством. Это представляет собой важный результат, поскольку позволяет понижать порядок дифференциальных сигма-модельных полевых уравнений второго порядка в частных производных, которые являются важным элементом в супергравитации и теории струн, а также в квантовой теории поля.

С точки зрения анализа другой существенный результат настоящей работы можно рассматривать как порождение расширенной ли-алгебраической структуры исходя из разрешимой подалгебры Ли в алгебре Ли. Рассуждения из раздела 2 легко обобщить, и на основе полного и формального доказательства из раздела 2 можно утверждать, что каждая подалгебра борелевской подалгебры алгебры Ли находится внутри другой алгебры Ли с удвоенной размерностью.

\section{Список литературы}

[1] E. Cremmer, B. Julia, H. Lü, C. N. Pope, Nucl. Phys. B, 523:1-2 (1998), 73-144; arXiv: hep-th/9710119.

[2] E. Cremmer, B. Julia, H. Lü, C. N. Pope, Nucl. Phys. B, 535:1-2 (1998), 242-292; arXiv: hep-th/9806106.

[3] N.T. Yılmaz, Nucl. Phys. B, 664:1-2 (2003), 357-370; arXiv: hep-th/0301236.

[4] N. T. Yılmaz, Nucl. Phys. B, 675:1-2 (2003), 122-138; arXiv: hep-th/0407006.

[5] A. Keurentjes, Nucl. Phys. B, 658:3 (2003), 303-347; arXiv: hep-th/0210178.

[6] A. Keurentjes, Nucl. Phys. B, 658:3 (2003), 348-372; arXiv: hep-th/0212024. 